\title{
Case report: Amyand hernia reincarnate and the utility of computed tomography
}

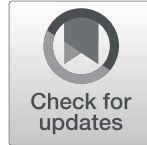

Joshua Fields ${ }^{1}$, Kristen Quinn ${ }^{2}$, Laura Hollinger ${ }^{2}$ and Richard Jones ${ }^{3^{*}}$ (D)

\begin{abstract}
Background: An Amyand hernia is a rare condition in which the vermiform appendix follows the bowel as it bulges through the inguinal canal. Originally described by Claudius Amyand in 1735 in an 11-year-old boy who had a pin in the appendix (Philos Trans R Soc Lond. 1735;39:329-36), we describe here the first case with CT depicting all components of Dr. Amyand's original case.

Case presentation: A 10-year-old boy presented with right lower quadrant and groin pain. CT imaging revealed a retained metallic foreign body perforating the appendix, acute appendicitis, and an Amyand hernia. A laparoscopic attempt at reduction failed due to tethering by the needle and inflammation of the hernia sac, requiring an open inguinal exploration and excision of the hernia sac. The patient was discharged on postoperative day 2 with plans for delayed hernia repair.

Conclusions: $\mathrm{CT}$ is a crucial diagnostic test for surgical decision-making in Amyand hernias. In this case, it accurately classified the hernia and led to anticipation of unsuccessful laparoscopic hernia reduction. Management in the setting of foreign body and perforation includes appendectomy and tissue herniorrhaphy with delayed formal inguinal hernia repair after inflammation has subsided.
\end{abstract}

Keywords: Amyand hernia, Needle, Appendix, Computed tomography, Case report

\section{Background}

Inguinal hernias account for $75 \%$ of abdominal wall hernias [1]. When the appendix is incarcerated in the inguinal sac, this is known as Amyand's hernia and can present with or without acute appendicitis [2]. Preoperative imaging studies help with operative planning. US, $\mathrm{CT}$, and MRI can be used to image the inguinal canal and appendix. Imaging studies guide the surgical approach and mitigate intraoperative findings. CT and US are the most commonly used imaging modalities used to diagnose acute appendicitis in pediatric patients today. While US is less costly and has no ionizing radiation, $\mathrm{CT}$ is more accurate for diagnosis of perforations and other complications seen in one-third of pediatric

\footnotetext{
* Correspondence: jonerich@musc.edu

${ }^{3}$ Department of Radiology and Radiological Sciences, Division of Pediatrics, Medical University of South Carolina, 96 Jonathan Lucas St CSB HE210C MSC 323, Charleston, SC 29425, USA

Full list of author information is available at the end of the article
}

patients [3]. MRI is less commonly used for the diagnosis of appendicitis due to availability, cost, and possible need for sedation. MRI has similar sensitivity and specificity to CT and finds an explanation for abdominal pain other than appendicitis in $20 \%$ of suspected cases [4].

An Amyand hernia is a rare form of an inguinal hernia $(<1 \%)$ in which the appendix is incarcerated within the inguinal canal. The incarcerated appendix can present with or without appendicitis. Less than 1\% of Amyand hernias present with acute appendicitis. Losanoff and Basson's criteria is classification system developed for adults with four types; type 1 is a normal appendix in a hernia sac; type 2 is a hernia with acute appendicitis; type 3 is a hernia with acute appendicitis and abdominal sepsis; type 4 is acute appendicitis with related or unrelated abdominal pathology [5].

The first case was described by Claudius Amyand in 1735 when he found the appendix within the hernia sac while repairing an 11-year-old boy's hernia [6]. Finding a 
pin within the appendix within an inguinal hernia is remarkably rare, almost once every century, as there have been only three previously described (Amyand 1735, Hall 1886, Llullaku 2006) [7]. This is the first case in which CT was used to make the diagnosis and was an integral part of identifying this rare phenomenon as no history of foreign body ingestion could be elicited.

\section{Case presentation}

A 10-year-old boy presented with 3 days of worsening right-lower quadrant abdominal pain and swelling of the right groin. He had no prior medical history or surgery. He was first seen by his pediatrician who then referred him to an emergency department. In the ED, the patient was afebrile, normotensive, not tachycardic, and endorsing pain. On exam, abdomen was soft and bowel sounds were present. There was tenderness to palpation and guarding. He exhibited a nonreducible right inguinal mass with mild erythema that was extremely tender to palpation (Fig. 1). He received fluid resuscitation, antibiotics, and a CT of the abdomen and pelvis. This demonstrated the appendix passing lateral to the inferior epigastric vessels into the right inguinal canal. The appendix became progressively thicker and surrounded with more fluid as it passed into the hernia $(8 \mathrm{~mm}$ diameter by the cecum, $14 \mathrm{~mm}$ in the hernia sac). The hernia sac was $2.5 \times 2.5 \mathrm{~cm}$ in short axis and $6 \mathrm{~cm}$ long. There was inflammatory fat stranding and thickening of the adjacent subcutaneous fat and scrotum and a small right hydrocele. The right testis was normal. One end of a

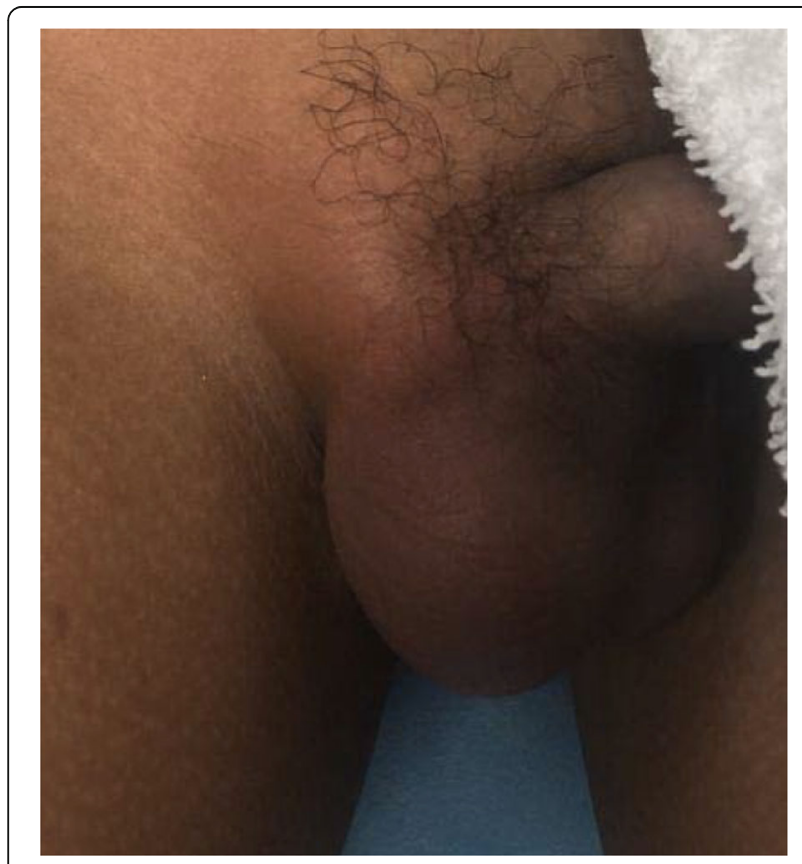

Fig. 1 Patient presented with nonreducible, erythematous right inguinal mass extreme tenderness to palpation 2.4-cm linear metallic foreign body was inside the appendix inferiorly, but the superior end pierced the posterior wall of the appendix (Figs. 2 and 3). This was interpreted as an Amyand hernia with appendicitis and perforation, type 2 according to Losanoff and Basson. There was no proximally dilated bowel, appendicolith, air within the hernia sac or peritoneal cavity, localized abscess, or fistula to the skin. Despite the urgency of this case due to possibility of bowel ischemia given 3 days of abdominal pain, CT caused no delay in management of this patient. In fact, $\mathrm{CT}$ allowed clear communication of the diagnosis between medical centers as the images were viewed on PACS used by physicians in both centers.

The patient was then transferred to our medical center for definitive surgical management as no qualified pediatric surgeon was available at the outside institution. On evaluation, his right groin bulge was not reducible and pain was persistent. His vital signs remained stable. He was taken to the operating room urgently for laparoscopic appendectomy and inguinal hernia exploration. Initially, we performed a laparoscopic attempt at reduction and dissected the proximal appendix away from the indirect hernia sac, taking care to preserve the vas deferens and testicular vessels (Fig. 4a, b). We were unable to completely reduce the appendix from the inguinal canal despite internal traction with laparoscopic graspers and external groin pressure. We therefore ligated the appendiceal base and mesentery from the cecum laparoscopically and performed an open inguinal exploration. After elevating the spermatic cord and hernia sac, we separated the distal appendix from within the hernia sac and found a hemming needle extruding from its lumen (Fig. 5a, b). The specimens were removed (Fig. 5c), and the testis and its blood supply were found to be intact. We copiously irrigated and then closed the inguinal canal in a purse-string manner to approximate a high ligation. The patient tolerated the procedure well and was discharged home on postoperative day 2, with follow-up planned for right inguinal hernia repair. The patient denies ingesting the hemming needle. Nine months after surgery, the patient and his family did not return for a follow-up appointment.

\section{Discussion}

Historically, discovering the appendix in the inguinal sac was an incidental intraoperative finding, but this has become less common with increased use of CT. In our case, CT prepared the surgical team to (1) expect a sharp in the field and (2) expect a difficult laparoscopic reduction of the appendix from the hernia sac-the tip of the hemming needle passed through the wall of the appendix into the hernia sac, anchoring it within the sac. Periappendiceal inflammation also produced a tethering effect on the hernia sac. Perhaps the relatively larger size 


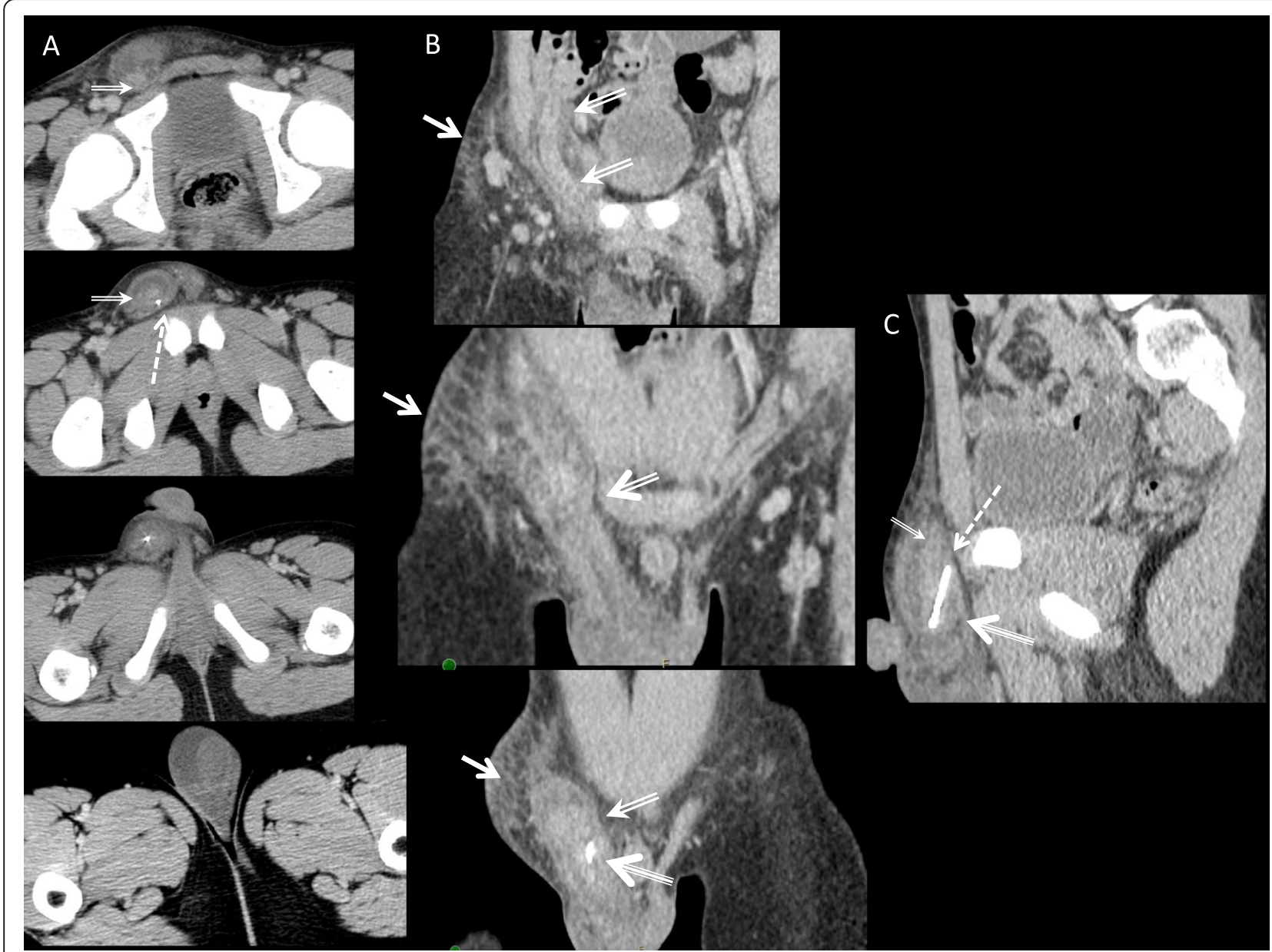

Fig. 2 a Successive axial CT images from the inguinal canal to the scrotum show the needle-filled appendix becoming thicker inferiorly within the hernia sac, fluid within the sac, and the needle tip passing posterior and outside of the appendiceal lumen. The most inferior slice shows the testis and small hydrocele. $\mathbf{b}$ Successive coronal reformatted $\mathrm{CT}$ images show the appendix connecting to the cecal tip and passing into the inguinal canal with surrounding subcutaneous fat stranding. c Sagittal image of the right inguinal canal showing the superior end of the needle passing through the posterior wall of the thickened appendix, with fluid in the inguinal canal. Double arrow, appendix; triple arrow, pin; dashed arrow, point where pin passes through the appendiceal wall; solid arrow, subcutaneous swelling in the groin

of the tip of the appendix relative to the thinner body of the appendix in the inguinal canal predisposed our patient to a difficult reduction requiring open exploration and repair. CT accurately depicted all these features and can do so for all types of Losanoff and Basson's Amyand hernia classification system.

Appendectomy-whether laparoscopic, through the hernia sac, or via laparotomy-and concurrent or delayed inguinal hernia repair is the treatment for Amyand hernia with appendicitis [2, 5]. Open inguinal exploration may be needed to completely reduce the appendix if laparoscopic attempts fail. Inguinal hernia repair at the time of this operation was contraindicated due to the acute inflammation and foreign body and risk for subsequent wound infection.
Children have a higher incidence of foreign bodies in the appendix due to the higher incidence of accidental ingestion. The majority of foreign bodies that are ingested will pass without problem through the GI tract. Less than $1 \%$ of these foreign bodies become entrapped in the appendix due to gravity and its position to the cecum while both upright and supine $[8,9]$. When a foreign body is found within the appendix, sharp objects, like our patient's needle, are more likely to cause perforation than blunt objects [9]. The sharp objects tend to cause rupture within days, while the blunt objects can present with recurrent abdominal pain, acute appendicitis, or asymptomatically [10]. When managing a patient with suspected ingestion of a foreign body, serial physical exams and radiographs should be taken. If not 


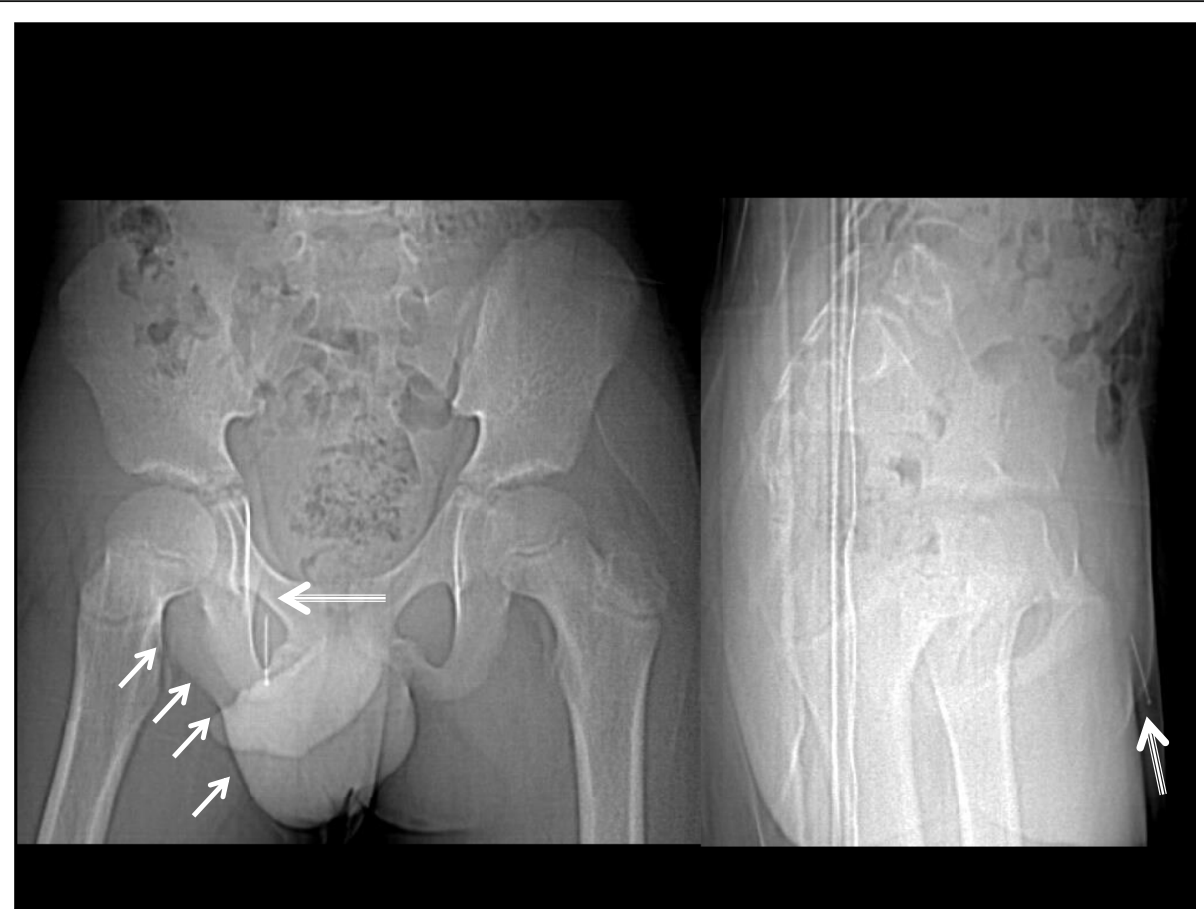

Fig. 3 Frontal and lateral scout CT images of the pelvis showing the pin in the right inguinal canal (triple arrow) with adjacent swelling in the groin and right hemiscrotum (single arrows)

progressing and possibly entrapped, CT should be used to localize the object in the appendix [8].

Acute appendicitis has a relatively high incidence in pediatric patients, but diagnosis can be delayed because an estimated one-third of children present abnormally [3]. Perforation is more frequent in children than adults, so accurate imaging needs to be available for surgical management. CT is a valuable imaging modality to diagnose this condition as it allows for better visualization of a retrocecal appendix and is able to delineate extent of inflammation and abscess formation. It also has less interference from air in the bowel, adiposity, and operator error than US [3]. Our patient's appendix was likely chronically herniated in the relatively fixed shape of the inguinal canal, giving it a small diameter in the canal and a more bulbous tip in the superior hemiscrotum. This anatomic spatial constraint may have made our patient unable to expel a foreign body from the appendix once it reached the inguinal canal, effectively predisposing him to perforation and appendicitis.

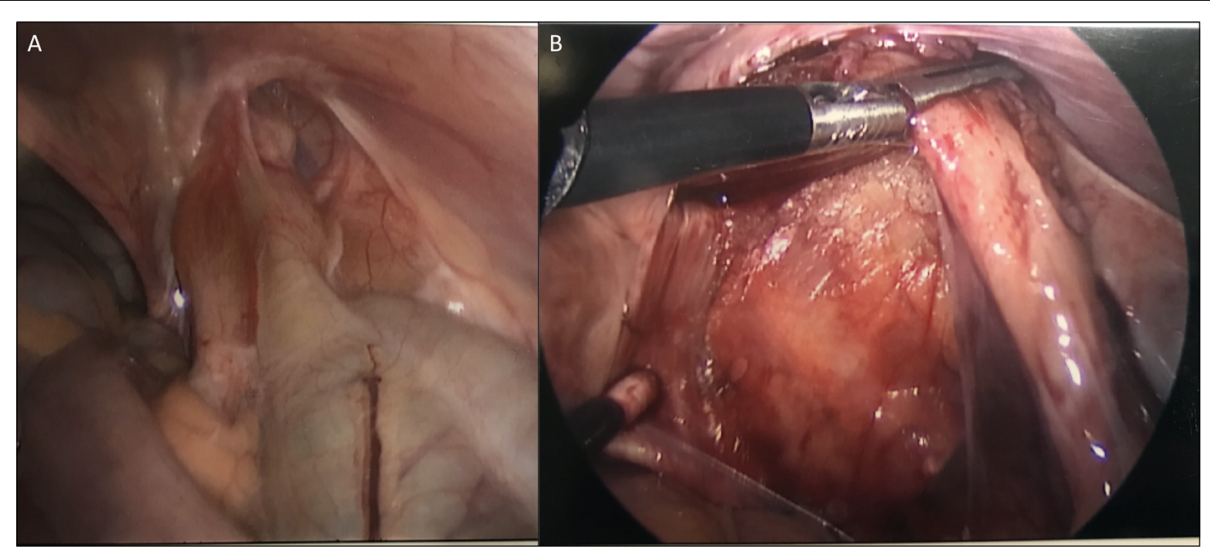

Fig. 4 Laparoscopic images. a Of appendix in right inguinal canal. b Laparoscopic image of dissection of appendix out of canal with vas deferens and testicular vessels preserved 


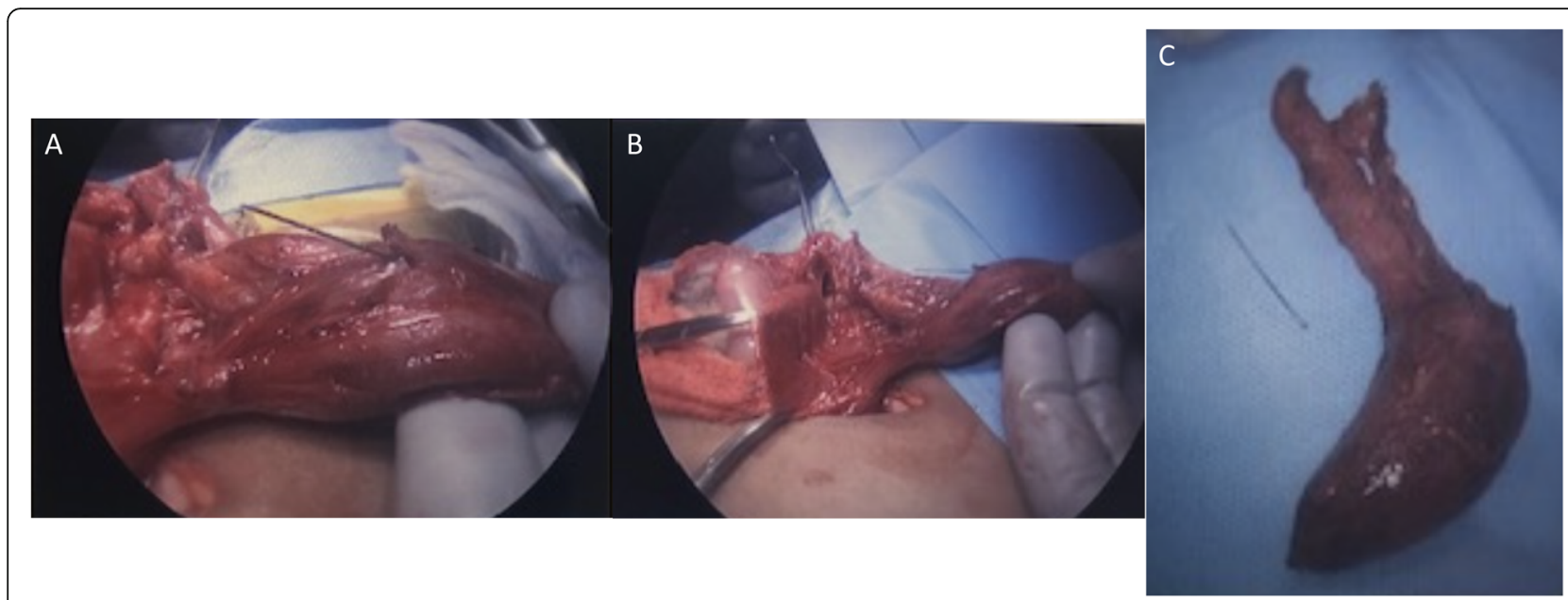

Fig. 5 Surgical removal of incarcerated appendix. a Needle protruding from lumen. b Appendix containing needle to the right, testis to left. c Appendix with needle removed

\section{Conclusion}

A high index of suspicion and CT imaging assisted with accurate diagnosis and treatment of our patient's Amyand hernia. Management in the setting of foreign body and perforation includes appendectomy and tissue herniorrhaphy with delayed formal inguinal hernia repair after inflammation has subsided. With $\mathrm{CT}$, this rare form of hernia can be diagnosed before surgery to allow safer preoperative planning and intraoperative decisionmaking. Our 11-year-old patient also had a pin in his appendix, just like Hanvil Anderson, the 10-year-old treated at St. George's hospital by Dr. Amyand in London in 1735.

\section{Abbreviations}

CT: Computed tomography; US: Ultrasound; MRI: Magnetic resonance imaging; ED: Emergency department

\section{Acknowledgements}

N/A

\section{Authors' contributions}

JF: wrote and compiled the manuscript and references. LH and KQ: edited the manuscript and provided clinical and intraoperative descriptions and images. RJ: edited the manuscript, provided CT images and descriptions, prepared the manuscript for submission, and submitted the manuscript. All authors have read and approved the submitted manuscript.

\section{Funding}

None.

\section{Availability of data and materials}

Data sharing is not applicable to this article as no datasets were generated or analyzed during the current study.

\section{Ethics approval and consent to participate}

This case is considered exempt from review by Medical University of South Carolina's Institutional Review Board because it does not constitute research (official policy attached, but also copied here). CT and clinical images were submitted as part of this case report but all the identifying information has been removed.
Per HRPP 3.7 (III A): it is the policy that the publication of case reports of three or fewer patients is NOT considered human-subject research and does NOT typically require IRB review and approval because case reporting on a small series of patients does not involve the formulation of a research hypothesis that is subsequently investigated prospectively and systematically for publication or presentation. Therefore, three or less case is not considered research but rather a clinical exercise.

\section{Consent for publication}

Due to the novelty of this case, verbal consent for publication was obtained from the patient and his mother during the perioperative period as there was no available written consent form on hand at the time of surgery. The patient and his family have not been able to be reached by phone or email since surgery and have not followed up for a postoperative visit at this time ( 9 months postop). His pediatrician has not been able to contact the patient or mother. The patient may have moved to a new home or location but whereabouts are currently unknown. As such, verbal, but not written consent to publish this information has been obtained.

\section{Competing interests}

The authors declare that they have no competing interests.

\section{Author details}

${ }^{1}$ Medical University of South Carolina, 96 Jonathan Lucas St CSB 601, Charleston, SC 29425, USA. ${ }^{2}$ Department of Surgery, Division of Pediatric Surgery, Medical University of South Carolina, 96 Jonathan Lucas St CSB 417, Charleston, SC 29425, USA. ${ }^{3}$ Department of Radiology and Radiological Sciences, Division of Pediatrics, Medical University of South Carolina, 96 Jonathan Lucas St CSB HE210C MSC 323, Charleston, SC 29425, USA.

Received: 31 May 2020 Accepted: 27 July 2020

Published online: 15 October 2020

\section{References}

1. Jenkins JT, O'Dwyer PJ. Inguinal hernias. BMJ. 2008;336:269-72.

2. Singal R, Gupta S. "Amyand's Hernia"- pathophysiology, role of investigations and treatment. Maedica. 2011;6(4):321-7.

3. Doria AS. Optimizing role of imagine in appendicitis. Pediatr Radiol. 2009; 39(Suppl 2):S144-8.

4. Moore MM, Kulaylat AN, Hollenbeak CS, Engbrecht BW, Dillman JR, Methratta ST. Magnetic resonance imaging in pediatric appendicitis: a systematic review. Pediatr Radiol. 2016;46:928-39.

5. Losanoff J, Basson M. Amyand Hernia: what lies beneath - a proposed classification scheme to determine management. Am Surg. 2007;73:128890. 
6. Amyand C. VII. Of an inguinal rupture, with a hemming needle in the appendix coeci, incrusted with stone; and some observations on wounds in the guts. Philos Trans R Soc Lond. 1735;39:329-36.

7. Llullaku SS, Hyseni NS, Kelmendi BZ, Jashari HJ, Hasani AS. A hemming needle in appendix within Amyand's hernia in a six-year-old boy: case report and review of literature. World Journal of Emergency Surgery. 2010;5: 14.

8. Hines JJ, Paek GK, Lee P, Wu L, Katz DS. Beyond appendicitis; radiologic review of unusual and rare pathology of the appendix. Abdominal Radiology. 2016;41:568-81.

9. Packard E, Groff A, Shahid Z, Sahu N, Jain R. A 'Bit' of appendicitis: a case of a foreign object in the adult appendix. Cureus. 2019;11(5):e4751. https://doi. org/10.7759/cureus.4751.

10. Hartin CW, Lau ST, Caty MG. Metallic foreign body in the appendix of a 3year-old boy. J Pediatr Surg. 2008;43:2106-8.

\section{Publisher's Note}

Springer Nature remains neutral with regard to jurisdictional claims in published maps and institutional affiliations.

\section{Submit your manuscript to a SpringerOpen ${ }^{\circ}$ journal and benefit from:}

- Convenient online submission

- Rigorous peer review

- Open access: articles freely available online

- High visibility within the field

- Retaining the copyright to your article

Submit your next manuscript at $\boldsymbol{\wedge}$ springeropen.com 